\title{
Pasteurella Multocida Subdural Empyema: A Case Report
}

\author{
M.I. KHAN and R. CHAN
}

SUMMARY: The first case of Pasteurella Multocida subdural empyema is reported. This was secondary to hematogenous spread from the pharynx to a chronic subdural hematoma. Positive cultures were obtained from the primary source, the blood and the subdural space. The patient was discharged with no neurological deficit following surgical drainage and parenteral antibiotics. The incidence, predisposing factors, clinical features, bacteriology. diagnostic procedures, treatment and mortality of subdural empyema are briefly discussed.

RÉSUMÉ: Nouns rapportons le premier cas d'empyème sousdural dú au Pasteurella Multocida. Celui-ci est secondaire à une diffusion hématogène vers un hématome sousdural chronique à partir cu pharynx. Des cultures positives furent obtenues de la source primaire, du sang et de l'espace sousdural. Après un drainage chirurgical et des antibiotiques parentéraux. le patient fut libéré sans déficit neurologique. Nous discutons l'incidence, les facteurs prédisposants, les aspects cliniques, la bactériologie. les procédures diagnostiques, le traitement et la mortalité de l'empyème sousdural.

From the Department of Clinical Neurological Sciences, Division of Neurosurgery, University of Saskatchewan, College of Medicine, Saskatoon, Saskatchewan.

Reprint requests to: Dr. M.I. Khan. Dept. of Clinical Neurological Sciences, University Hospital, Saskatoon. Saskatchewan, S7N 0X0. Canada.

\section{INTRODUCTION}

Pasteurella Mutltocida is a nonmotile, gram negative, pleomorphic, bipolar-staining coccobacillus (Johnson \& Rumans, 1977). It differs from other gram-negative organisms in its uniform exquisite sensitivity to penicillin and ampicillin (Swartz \& Kunz, 1959). There are reports noting the common organisms found in subdural empyema (Bandari \& Sarkari, 1970; Botterell \& Drake, 1952; Coonrod \& Dans, 1972; Farmer \& Wise, 1973). No previous case of subdural empyema due to Pasteurella Multocida infection has been reported in the English literature. In addition, secondary contamination of a chronic subdural hematoma by hematogenous spread is rare.

\section{CASE REPORT}

A 62 year old chronic alcoholic male patient was admitted to the University Hospital in January, 1980. He had been complaining of headaches and loss of appetite for about 3 or 4 weeks. He decreased his alcohol intake and began to sleep more than usual. There was no history of recent head injury. Three days before admission he became drowsy and when roused was confused and complained of severe headache. On the day of admission, his wife found him lying on the floor of the bathroom.

In April, 1979 he was found to have an epidermoid carcinoma of the dorsum to his tongue and this was treated by external radiation and local radium implantation. In September, 1979 follow up examination revealed evidence of esophageal metastasis which were treated by radiation and chemotherapy. There was no previous history of sinusitis, otitis media or recent infection.

On admission the patient was acutely ill. He was confused, lethargic and shaky. He appeared mildly dehydrated and had a temperature of $39.4^{\circ} \mathrm{C}$. The heart rate was 110 per minute and respiratory rate 28 per minute. He had nuchal rigidity and positive Kernig's sign. The neurological examination revealed no other abnormality. His breath had a foul odor and the orophaynx appeared injected with a few yellowish appearing plaques.

There was peripheral leukocytosis with a shift to the left. Random blood glucose was $216 \mathrm{mg} / \%$. Lumbar puncture was performed with an opening pressure of $240 \mathrm{~mm} / \mathrm{H}_{2} \mathrm{O}$. The CSF was slightly turbid and contained $491 \mathrm{WBC} / \mathrm{mm}^{3}$ with $72 \%$ neutrophils and $24 \%$ monocytes, glucose, $94 \mathrm{mg} / \%$ and protein $143 \mathrm{mg} / \%$. Gram stain on the CSF revealed no organism and culture was negative. However, cultures from both the pharynx and the blood were later found to be positive for Pasteurella Multocida. Plain x-ray films of the skull were normal. A CT scan examination without contrast showed a left frontoparietal subdural fluid collection (Fig. 1).

Four hours after lumbar puncture, the neurological examination revealed a comatose man with nuchal rigidity and occasional spontaneous decerebrate posturing. There was no papilledema and the pupils were $3 \mathrm{~mm}$ bilaterally and reacted slugishly to light. He responded to deep painful stimuli by decerebrate movements in his limbs. The tendon reflexes were hyperactive on the right side with a positive Babinski sign. The heart rate was 70 per minute and the respiratory rate was 30 per minute.

He was given 3 grams ampicillin and started on $500 \mathrm{cc}$ of $20 \%$ mannitol intravenously. He was then taken to the operating room where two burr holes were placed, one over the left 


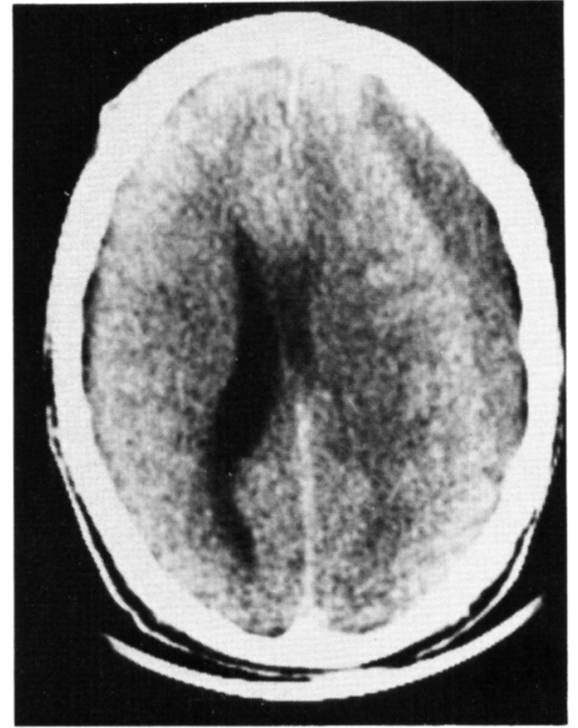

Figure I - Unenhanced pre-operative CT Scan showing a left fronto-parietal subdural fluid collection.

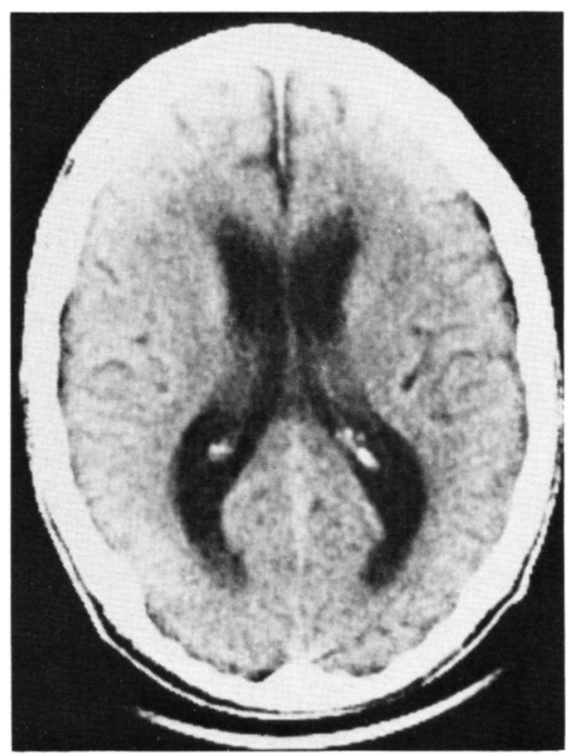

Figure 2 - CT Scan 2 weeks postoperative

frontal and the other over the left temporoparietal area. Upon opening the dura in the left frontal area, dark yellowish liquid, typical of a chronic subdural hematoma with a thick membranous sac, was encountered while thick purulent chocolate coloured fluid came out from the temporoparietal opening. The subdural space was in continuation without obvious multiloculated membranes. It was thoroughly irrigated with normal saline and bacitracin solutions. Two rubber catheter drains were left in the subdural space for subsequent drainage and were removed 48 hours later. Gram stain of the fluid removed from the subdural space showed gramnegative rods, and culture grew Pasteurella Multocida organisms.

The patient developed focal right sided seizures on the 2 nd postoperative day in spite of prophylactic phenytoin theraphy. These progressed to status epilepticus which was finally controlled by phenobarbital, diazepam and paraldehyde. He remained in a critical state during the first 4 postoperative days and required mechanical ventilation and frequent suctioning. He subsequently improved rapidly and was maintained on intravenous ampicillin for about 3 weeks. A repeat CT scan, two weeks after surgery, revealed complete resolution of the subdural empyema. (Fig. 2) $\mathrm{He}$ was discharged one month later on phenytoin without any neurological deficit.

\section{DISCUSSION}

The presence of a subdural empyema represents a neurosurgical emergency. It is probably not well recognized as is reflected by frequent late referral, wrong diagnosis and high mortality ranging between 15 and $43 \%$ (Bhandari \& Sarkari, 1970; Coonrod \& Dans, 1972: Farmer \& Wise, 1973; Kaufman et al., 1975). This disease accounts for 13 to $20 \%$ of all intracranial abscesses (Galbraith \& Barr, 1974; Weinman \& Samarsinghe, 1972). Males are affected three times as commonly as females (Kaufman et al., 1975; Weinman \& Samarsinghe, 1972; Glasauer et al., 1978). Predisposing factors in decreasing order of frequency are: sinusitis, especially frontal, otitis media or mastoiditis, meningitis, trauma and postoperative complications. Hematogenous spread is relatively uncommon. In particular, only a few cases secondary to bacteremic seeding of a chronic subdural hematoma have been reported (Coonrod \& Dans, 1972; Genest et al., 1963; Ferguson \& Gotoff, 1977).

Typically, subdural empyema is found in young men between the ages of
10 to 40 years. There is often a history of sinusitis and otitis media. Signs of systemic infection including fever, chills and mental changes precede cerebral dysfunction manifested by local or generalized seizures, hemiparesis, decreasing level of consciousness, dysphasia with involvement of the dominant hemisphere, visual and sensory disturbances, pupillary change, coma and finally death (Bhandari \& Sarkari, 1970). Symptoms of increased intracranial prissure, headache, nausea and vomiting are usually present but, papilledema is not common. Signs of meningeal irritation may be present. The most frequent offending organism is streptococcus followed by staphylococcus (Galbraith \& Barr, 1974; LeBeau et al., 1973). Skull films usually reveal clouding of the involved sinuses with pineal shift or suture diastasis in infants. Lumbar puncture usually demonatrates pleocytosis with normal glucose value, negative gram stain and culture (Coonrod \& Dans, 1972; Kubik \& Adams, 1943; Weinman \& Samarsinghe, 1972). The danger of lumbar puncture in the presence of focal neurological findings despite lack of papilledema has been stressed previously (Galbraith \& Barr, 1974; Glasauer et al., 1978; Kaufman et al., 1975). The patient presented here deteriorated rapidly after the lumbar puncture. Electroencephalogram is seldom of localizing value in subdural empyema. Isotope brain scan is limited in diagnostic usefulness (Glasauer et al., 1978). Cerebral angiography is considered the procedure of choice by many authors (Bhandari \& Sarkari, 1970; Coonrad \& Dans, 1972; Kaufman et al,, 1975; Weinman \& Samarsinghe, 1972). The "S" pattern found in parafalcine subdural empyema has been well documented. Subdural tap is usually diagnostic in pediatric cases. The CT scan examination offers a quick, non-invasive and accurate localizing method of diagnosing subdural fluid collection. However, it was not possible to differentiate a chronic subdural hematoma from a subdural empyema in this patient by our plain CT head scan.

The majority of subdural empyemas are supratentorial and over the convexity, either unilateral or bilate- 
ral, or interhemispheric with parafalcine pus collection. Early surgical drainage and parenteral antibiotics are extremely important in the management of this disease. No significant difference in the outcome between groups treated by multiple burr holes, craniectomy or craniotomy was noted (Glasauer et al., 1978). The issue of local antibiotics is not settled (LeBeau et al., 1973). It is suggested that the instillation of penicillin locally may be epileptogenic. If used, less neurotoxic bacitracin or oxytetracycline is preferred. Intravenous antibiotics should be continued for three weeks or longer in cases of resistant organisms and associated osteomyelitis. Penicillin and /or chloramphenicol are the drugs of choice until the cultures and sensitivities are available. Prophylactic anticonvulsant theraphy is recommended both pre-operatively and postoperatively.

This is the first reported case of a subdural empyema with Pasteurella Multocida organisms. Positive cultures were obtained from the primary source (oropharynx), the blood and the subdural space. The Pasteurella Multocida organism is a common oral commensal of animals including cats and dogs and is, therefore, a characteristic pathogen following animal bites and scratches. However, this organism is also an occasional saphrophytic resident of the nasopharynx or upper respiratory tract of healthy man (Johnson \& Rumans, 1977). Although this patient had a dog, there was no history of bites or scratches. However, recent malignancy involving the tongue and esophagus as well as treatment with local radium implants, cobalt therapy and chemotherapy, probably reduced resistance to infection both locally and systemically.

\section{REFERENCES}

BHANDARI, Y.S.. SARKARI, N.B.S. (1970). Subdural Empyema. A review of 37 cases. J. Neurosurg 32: 35-39.

BOTTERELL. E.H.. DRAKE, C.G. (1952). Localized encephalitis, brain abscess and subdural empyema. J Neurosurg 9: 348-366.

COONROD, J.D., DANS, P.D. (1972). Subdural Empyema. Am. J. Med. 53: 85-91.

FARMER, T.W., WISE, G.R. (1973). Subdural empyema in infants, children and adults. Neurology 23: 254-261.

FERGUSON. L., GOTOFF. S.P. (1977). Subdural empyema in an infant due to group
B B-hemolytic Streptococcus. Am J Dis Child 131: 97.

GALBRAITH. J.G., BARR, V.W. (1974). Epidural abscess and subdural empyema. Adv Neurology 6: 257-267.

GENEST, A.S., BINGHAM, W.G., HAMILTON, R.D. (1963). Bilateral subdural empyema. Report of a case with arteriograms. J Neurosurg 20: 524-526.

GLASAUER, F.E., COOTS, D.. LEVY, L.F.. et al. (1978). Subdural empyema in Africans in Rhodesia. Neurosurg 3: 385.

JOHNSON, R.H., RUMANS, L.W. (1977). Unusual infections caused by Pasteurella Multocida. JAMA 237 (2): 146-147.

KAUFMAN, D.M., MILLER, M.H., STEIGBIGEL, N.H. (1975). Subdural empyema analysis of 17 recent cases and review of the literature. Medicine 54(6): 485.

KUBIK, C.S., ADAMS, R.D. (1943). Subdural empyema. Brain 66: 18-42.

LEBEAU. J., CREISSARD, P.. HARISPE. L., et al. (1973). Surgical treatment of brain abscesses and subdural empyema. J. Neurosurg. 38: 198-203.

MILDER, J.E., HALL, N.K., FINLEY, R.A. (1977). Pasteurella Multocida pneumonia and bacteremia. South Med. J. 70 (9): 11231124.

SWARTZ, M.N., KUNZ, L.J. (1959). Pasteurella Multocida infections in man. Report of two cases - meningitis and infected cat bite. N. Engl. J. Med. 261 (18): 889-893.

WEINMAN, D., SAMARASINGHE, H.H.R. (1972). Subdural empyema. Aust NZ Surg 41: $324-330$. 